\title{
Distribución y abundancia del zooplancton del complejo lagunar Chacahua- La Pastoría, Oaxaca, México
}

\section{Distribution and abundance of zooplankton of the lagoon system Chacahua- La Pastoria, Oaxaca, Mexico}

\author{
Benigno Pantaleón-López ${ }^{1}$, G. Aceves ${ }^{2}$ e Iván A. Castellanos ${ }^{3}$ \\ ${ }^{1}$ Centro Bachillerato Técnológico Industrial y de Servicios No. 231, Santa María, Huatulco, Oaxaca, \\ México \\ ${ }^{2}$ Universidad del Mar, Carr. a Zipolite Km 1.5, Puerto Angel, Oaxaca, México \\ ${ }^{3}$ El Colegio de la Frontera Sur-Chetumal, Av. Centenario Km 5.5, Chetumal, Q. Roo, México \\ Correspondencia: ivancast@ecosur-qroo.mx
}

\begin{abstract}
Resumen. Se estudió la comunidad zooplánctica del sistema lagunar Chacahua-La Pastoría, antes de la apertura de la barra arenosa de Chacahua por la Secretaría de Marina. Se identificaron 26 grupos de zooplancton provenientes de 5 muestreos realizados en Mayo, Octubre, Diciembre de 1996 y Febrero y Junio de 1997, en el área investigada. En la Pastoría se registraron 25 grupos y en Chacahua 19; de los 26 grupos determinados 18 fueron comunes en ambas lagunas. El grupo más abundante fue el de las larvas de braquiuros que representaron el $35.7 \%$ del total del zooplancton, fueron seguidos por las larvas y huevos de peces con 22.74 y $15.26 \%$, respectivamente. Los máximos valores promedio de abundancia se observaron durante el periodo de sequía, en las zonas aledañas al canal de intercomunicación de ambas lagunas, así como en las cercanías del canal de comunicación con el mar. En Chacahua se observaron las mayores densidades en Junio, 1997 y en La Pastoría en Diciembre, 1996. En general en la laguna La Pastoría la riqueza de grupos fue mayor. La composición de la comunidad zooplánctica está condicionada por la salinidad y la tasa de intercambio de agua entre la laguna y el mar.
\end{abstract}

Palabras clave: Zooplancton, Oaxaca, sistema lagunar

\begin{abstract}
The zooplankton community of the lagoon system Chacahua-La Pastoría was studied before the opening of the sandy bar of Chacahua by the Secretaría de Marina. A total of 26 groups of zooplankton were identified at the lagoon complex Chacahua-La Pastoria made during five samplings in May, October and December, 1996 and February and June, 1997. In La Pastoria were recorded 25 groups and 19 in Chacahua. From 26 groups recorded, 18 were common in both lagoons. Braquiurid larvae were the most abundant group, and represent $35.7 \%$ of total zooplankton, followed by fish larvae and eggs with 22.74 and $15.26 \%$, respectively. The maximum mean values of abundance were observed in the dry period in the adjacent zones to the channel of intercommunication of both lagoons, as well as in the neighborhood of the communication channel with the sea. In Chacahua the highest values were observed in June, 1997 and in La Pastoría in December, 1996. In general the group richness was greater in La Pastoría lagoon. Zooplanktic community composition was conditioned for the salinity and water interchange rate between the sea and the lagoon.
\end{abstract}

Key words: Zooplankton, Oaxaca, lagunar system

\section{Introducción}

Los sistemas lagunares son de gran importancia ya que presentan una alta tasa de producción primaria y secundaria, además de ser zona de protección, reproducción, crecimiento y alimentación para una gran cantidad de especies marinas, estuarinas y dulceacuícolas (YañezArancibia, 1986). Estas características de las lagunas favorecen una mayor diversidad de organismos meropláncticos en la comunidad del zooplancton, en comparación con el holoplancton compuesto por escasas especies (Odum, 1972).

El zooplancton comprende una gran variedad de organismos que pueden considerarse como indicadores naturales de masas de agua y contaminación y también proporcionan información sobre las características del medio. Además sirven como base para programar acciones tendientes a eliminar o minimizar los impactos adversos en las lagunas; además los huevos y larvas de peces proveen información de cuándo y dónde se concentran los peces, permitiendo una mejor planeación para la captura de especies de importancia económica (Smith y Richardson, 1979; Tait, 1987).

Los estudios sobre zooplancton de las lagunas del Oaxaca son muy escasos. La mayor parte de la información se encuentra en resúmenes e informes internos, por consiguiente no se puede acceder a mayor información. Entre ellos está el de Teodoro y Ortíz (1990) quienes analizaron la composición y abundancia del zooplancton del complejo lagunar Chacahua-La Pastoría. En los trabajos realizados para el sistema lagunar Chacahua-La Pastoría por Contreras (1993), Contreras et al. (1994) y Rodríguez (1998), se señala que uno de los problemas que presenta este sistema es el azolvamiento, debido al desvío del cause de ríos para la agricultura. 
El Complejo Lagunar Chacahua-La Pastoría se ubica dentro del parque Nacional de Chacahua (Oaxaca) y se localiza entre los $15^{\circ} 57^{\prime}$ y los $16^{\circ} 01^{\prime}$ de latitud Norte y los $97^{\circ} 46^{\prime}$ y los $97^{\circ} 33^{\prime}$ de longitud Oeste (Sanay, 1997). La laguna de Chacahua tiene forma alargada, con una profundidad promedio de $1.8 \mathrm{~m}$ y una superficie aproximada de $600 \mathrm{Ha}$. Presenta una boca efímera, cerrada desde 1979 por una barra arenosa (Contreras et al., 1994). La laguna La Pastoría es la más grande, tiene una superficie de $1000 \mathrm{Ha}$ aproximadamente y presenta comunicación permanente con el mar en su parte oriental (Contreras et al., 1994; IMASA, 1995). Ambas lagunas están comunicadas por un canal denominado "El Corral", con orientación Oeste-Este y una longitud de tres kilómetros.

Este trabajo tiene como propósito fundamental analizar la composición, distribución y abundancia de la comunidad zooplánctica del sistema lagunar Chacahua-La Pastoría, a partir de recolectas realizadas entre 1996-1997, antes de la apertura de la barra arenosa de Chacahua por la Secretaría de Marina.

\section{Métodos}

El material zooplánctico proviene de recolectas efectuadas en 24 estaciones: 9 en la laguna de Chacahua y 15 en La Pastoría, en mayo, octubre y diciembre de 1996 y febrero y junio de 1997, cubriendo ampliamente el complejo lagunar (Fig. 1). Los sitios de muestreo para ambas lagunas se eligieron con base en un estudio prospectivo y tratando de cubrir las distintas áreas del complejo lagunar.

En todos los casos los arrastres de plancton fueron circulares en la capa 0-1 m de profundidad, con una duración de 3 minutos, a una velocidad aproximada de un nudo. Se empleó una red tipo Calcofi de $60 \mathrm{~cm}$ de diámetro y con abertura de malla de $0.5 \mathrm{~mm}$. A la boca de la red se le adaptó un flujómetro para estimar el volumen de agua filtrado en cada arrastre, fluctuando éste entre 111.09 y $284.63 \mathrm{~m}^{3}$. Las muestras fueron fijadas y preservadas en una solución de formaldehído al $4 \%$, amortiguada con borato de sodio. En cada sitio de muestreo se determinó la salinidad y la temperatura con un Hydrolab Surveyor 4, así como la profundidad del fondo.

La identificación de los organismos a nivel de grupo se realizó con base en los trabajos de: Tregouboff y Rose (1957), Smith (1977), Boltovskoy (1981) y Raymont (1983).

Las abundancias se agruparon en los siguientes intervalos de clase (num. de org/1000 $\mathrm{m}^{3}$ ), en escala logarítmica. Por ejemplo, el intervalo de 1-2 implica una densidad de 10-100 org $/ 1000 \mathrm{~m}^{3}$.

\section{Resultados}

Entre las dos lagunas se identificaron 26 grupos zoopláncticos. Destacaron por su abundancia relativa las larvas de braquiuros (35.7\%), las larvas de peces (22.74\%), los huevos de peces $(15.26 \%)$, las medusas $(7.43 \%)$ y los copépodos calanoides (5.87\%), constituyendo en conjunto el $87.01 \%$ del total de organismos recolectados. De los 26 grupos registrados
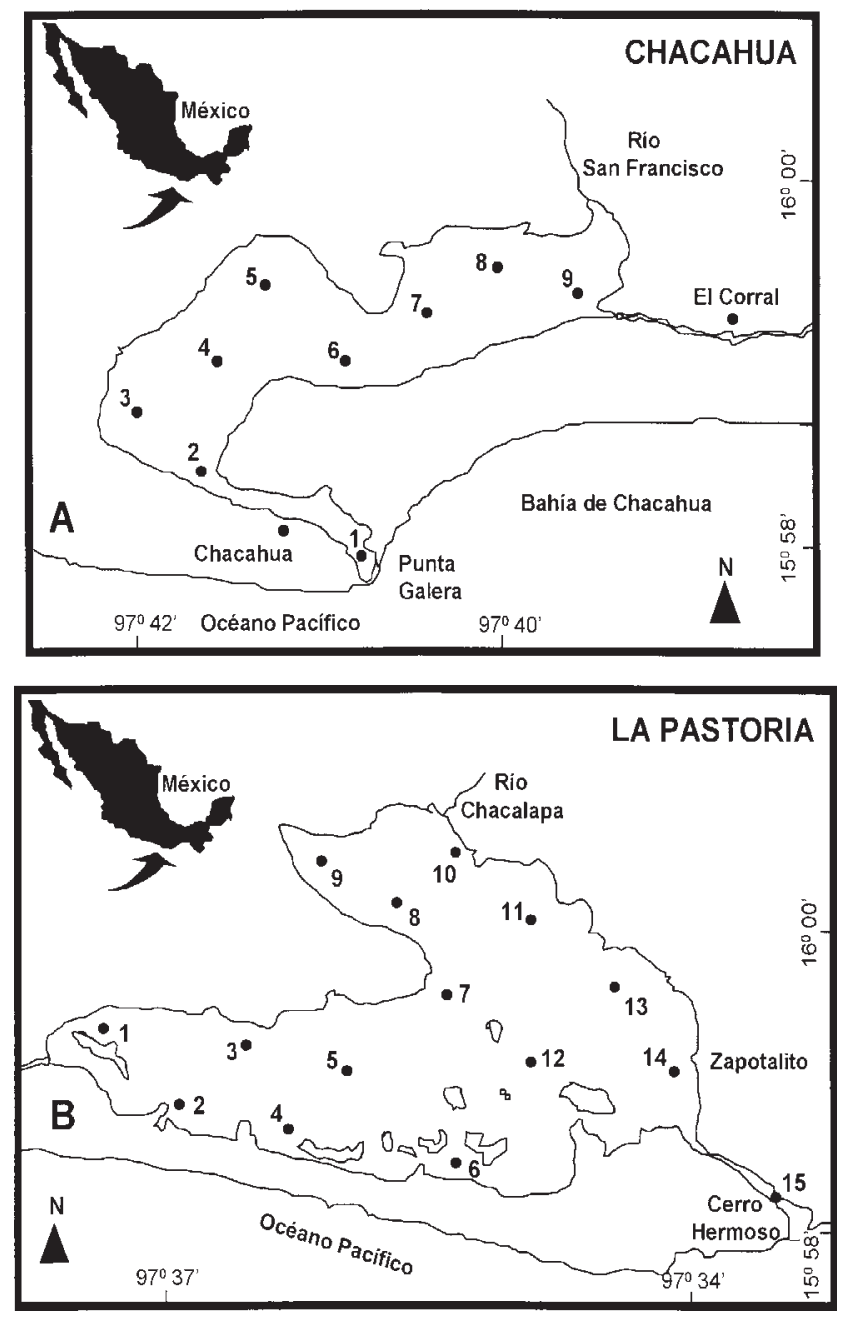

Figura 1. Area de estudio y ubicación de las estaciones de muestreo en las lagunas de A) Chacahua y B) La Pastoría, Oaxaca, México.

18 fueron comunes en ambas lagunas, siete se encontraron sólo en La Pastoría y uno en Chacahua.

Laguna Chacahua. La temperatura superficial promedio durante el período de estudio osciló entre los $28.9^{\circ} \mathrm{C}$ (Diciembre) y los $32.5^{\circ} \mathrm{C}$ (Junio); el valor mínimo se registró en Diciembre en la estación $1\left(27.6^{\circ} \mathrm{C}\right)$ y el máximo en Junio con $33^{\circ} \mathrm{C}$ en la estación 2 . La salinidad promedio osciló entre 1.3 y 65.3 PSU; los valores más bajos se registraron en las estaciones 3 y 7 en Octubre con 1.2 PSU y los más altos en las estaciones 7, 9 (65.6 PSU) y 8 (65.7 PSU) en Junio.

De los 19 grupos del zooplancton recolectados, los más abundantes fueron las larvas de braquiuros, las larvas y huevos de peces y las medusas, que en su conjunto constituyeron más del $98 \%$ del total. Los restantes 15 grupos representaron menos de $2 \%$ (Cuadro 1).

Las mayores abundancias se registraron en el mes de Junio de 1997 con 1,054,411 org/1000 m³ , siguiendo Febrero $\left(298,857 \mathrm{org} / 1000 \mathrm{~m}^{3}\right)$, Mayo (220,365 org $\left./ 1000 \mathrm{~m}^{3}\right)$, Diciembre y Octubre con 52,937 y $8,641 \mathrm{org} / 1000 \mathrm{~m}^{3}$, respectivamente.

El grupo más abundante fue el de las larvas de braquiuros (jaibas) que constituyó el 35.79\% del total de 
organismos recolectados. Su abundancia media fue de $117,061 \mathrm{org} / 1000 \mathrm{~m}^{3}$. Su máxima abundancia ocurrió en la estación 6 en Junio $\left(1,233,436\right.$ org/1000 $\left.\mathrm{m}^{3}\right)$ y la mínima en las estaciones 1 y 3 en Octubre con 295 y 322 org/1000 $\mathrm{m}^{3}$, respectivamente (Fig. 2). El mes de Junio registró el máximo promedio mensual con $500,910 \mathrm{org} / 1000 \mathrm{~m}^{3}$ ), mientras que el mínimo se observó en Octubre (5,384 org/ $1000 \mathrm{~m}^{3}$ ).

El segundo grupo más abundante fue el de las larvas de peces, el cual representó el $30.67 \%$ del total, con una abundancia promedio de $100,301 \mathrm{org} / 1000 \mathrm{~m}^{3}$. Las máximas y mínimas abundancias se observaron en las estaciones 5 (Junio) y 3 (Diciembre) con 880589 y $68 \mathrm{org} / 1000 \mathrm{~m}^{3}$, respectivamente (Fig. 3). En Octubre $\left(498 \mathrm{org} / 1000 \mathrm{~m}^{3}\right)$ se presentaron las menores abundancias promedio y las mayores en Junio con 351,674 org/1000 $\mathrm{m}^{3}$ (Cuadro 1).

Los huevos de peces constituyeron el $19.27 \%$ del total del zooplancton recolectado, con una abundancia media de $63,029 \mathrm{org} / 1000 \mathrm{~m}^{3}$. Se registraron los valores más bajos de densidad en Diciembre, en las estaciones 4 ( 55 org/1000 $\left.\mathrm{m}^{3}\right)$ y $8\left(48 \mathrm{org} / 1000 \mathrm{~m}^{3}\right)$. Los valores más altos se obtuvieron en Febrero, en la estación 5 con 481,584 org/1000 m³. Presentaron sus mayores abundancias promedio en Febrero $\left(247,121 \mathrm{org} / 1000 \mathrm{~m}^{3}\right)$ y las menores en Junio $(2,022 \mathrm{org} / 1000$ $\left.\mathrm{m}^{3}\right)$.

Las medusas ocuparon el cuarto lugar en abundancia con el $12.28 \%$ del total de organismos recolectados. Presentaron una abundancia media de 40,160 org/1000 m registrándose el valor más alto de densidad en Junio, en la estación $3(296,422$ org/1000 m³) y el más bajo en Mayo, en la estación 2, con $57 \mathrm{org} / 1000 \mathrm{~m}^{3}$. Otros grupos observados en la laguna de Chacahua fueron los misidáceos, los copépodos calanoides, los quetognatos, los poliquetos y las larvas de anomuros (Cuadro 1).

Las larvas de braquiuros se encontraron distribuidas ampliamente en Chacahua, registrándose generalmente las mayores densidades al norte y noreste de la laguna. Otros grupos que se encontraron distribuidos en toda la laguna fueron las larvas y los huevos de peces, aunque no mostraron un patrón de distribución definido. Durante Octubre y Diciembre las medusas no se observaron en Chacahua, mientras que en los restantes meses se encontraron distribuidas ampliamente en la laguna, presentando las mayores concentraciones al noreste de la misma.

Laguna La Pastoría. Los valores promedio de temperatura en la superficie variaron de 30.2 (Febrero) a $32.2^{\circ} \mathrm{C}$ (Octubre); en la estación 14, en Febrero se presentó la temperatura más baja con $25.5^{\circ} \mathrm{C}$ y la máxima se registró en Octubre con $32.8^{\circ} \mathrm{C}$ (en la estación 8). La salinidad promedio mensual varió entre los 6.2 PSU (Octubre) y 37.1 PSU (Junio); los valores más bajos se observaron en las estaciones 1 y 14 en octubre, con 5 y 5.7 PSU, respectivamente. La máxima salinidad se registró en Junio con 38.4 PSU, en la estación 1. Presentando una variación de más de 30 PSU.

En esta laguna se registraron 25 grupos. Las larvas de braquiuros, las larvas de peces, los copépodos calanoides

Cuadro 1. Abundancia promedio (org/1000 $\mathrm{m}^{3}$ ) y relativa (\%) de los grupos zoopláncticos encontrados en la laguna Chacachua, Oaxaca, durante el periodo de estudio.

\begin{tabular}{lrrrrrrr}
\hline Grupos & Mayo & Octubre & Diciembre & Febrero & Junio & $\begin{array}{c}\text { Abund. Prom. } \\
\text { org } / 1000 \mathrm{~m}^{3}\end{array}$ & $\begin{array}{c}\text { Abund. } \\
\text { Relativa } \%\end{array}$ \\
\hline Larvas de braquiuros & 32088 & 5384 & 32213 & 14709 & 500910 & 117061 & 35.79 \\
Larvas de peces & 125584 & 1561 & 498 & 22188 & 351674 & 100301 & 30.67 \\
Huevos de peces & 53465 & 0 & 12537 & 247121 & 2022 & 63029 & 19.27 \\
Medusas & 2050 & 0 & 0 & 759 & 197991 & 40160 & 12.28 \\
Misidáceos & 4118 & 596 & 5675 & 1839 & 1177 & 2681 & 0.82 \\
Copépodos calanoides & 2000 & 135 & 1960 & 10364 & 0 & 2892 & 0.88 \\
Larvas de anomuros & 391 & 0 & 0 & 622 & 534 & 309 & 0.09 \\
Poliquetos & 51 & 214 & 0 & 473 & 51 & 158 & 0.05 \\
Copépodos sifonostomatoides & 423 & 0 & 0 & 12 & 40 & 95 & 0.03 \\
Quetognatos & 105 & 0 & 6 & 449 & 0 & 112 & 0.03 \\
Ostrácodos & 0 & 371 & 0 & 17 & 0 & 78 & 0.02 \\
Anfípodos & 0 & 366 & 0 & 21 & 0 & 77 & 0.02 \\
Bivalvos & 6 & 0 & 0 & 120 & 0 & 25 & 0.01 \\
Carideos & 0 & 0 & 0 & 115 & 0 & 23 & 0.01 \\
Tanaidáceos & 66 & 0 & 0 & 0 & 8 & 15 & 0.005 \\
Branquiuros & 0 & 9 & 47 & 8 & 4 & 14 & 0.004 \\
Cirripedios & 0 & 0 & 0 & 39 & 0 & 8 & 0.002 \\
Gasterópodos & 17 & 0 & 0 & 0 & 0 & 3 & 0.001 \\
Peneidos & 0 & 7 & 0 & 0 & 0 & 1 & 0.0004 \\
\hline Total & 220365 & 8641 & 52937 & 298857 & 1054411 & 327042 & 100.00 \\
\hline
\end{tabular}




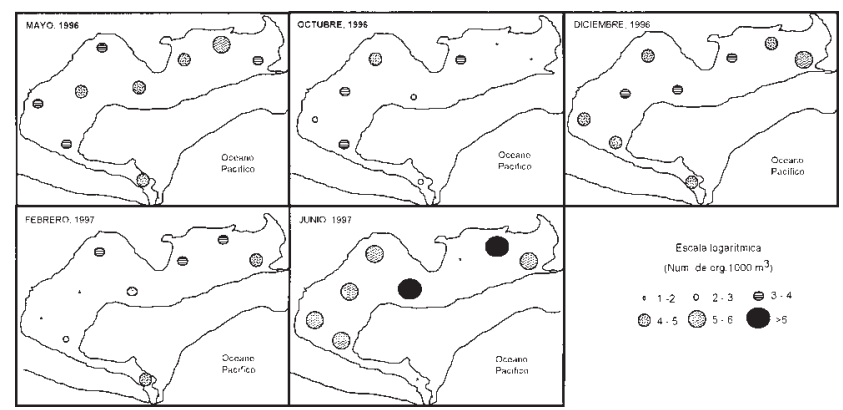

Figura 2. Variación espacio-temporal de la abundancia de larvas de braquiuros en la laguna de Chacahua (* estación sin organismos).

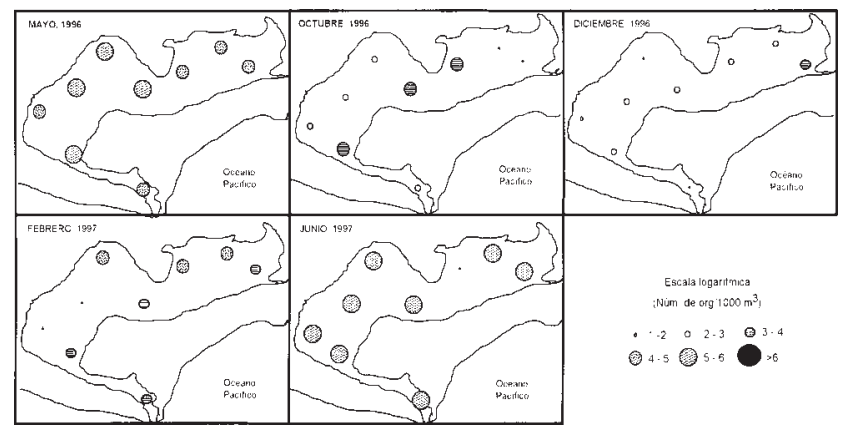

Figura 3. Variación espacio-temporal de la abundancia de larvas de peces en la laguna de Chacahua (* estación sin organismos).

y los huevos de peces representaron el $71 \%$ del total de los organismos recolectados (Cuadro 2).

Las máximas abundancias de organismos se presentaron en Diciembre de 1997 con 402,162 org/1000 $\mathrm{m}^{3}$, siguiendo Junio con $361,174 \mathrm{org} / 1000 \mathrm{~m}^{3}$, Mayo con 256,348 org $/ 1000 \mathrm{~m}^{3}$, Febrero y Octubre con 237,084 y $32,828 \mathrm{org} / 1000 \mathrm{~m}^{3}$, respectivamente.

El grupo más abundante fue el de las larvas de braquiuros, constituyendo el $35.58 \%$ del total de organismos recolectados; su abundancia promedio fue de $91,764 \mathrm{org} / 1000 \mathrm{~m}^{3}$. Su mínima abundancia ocurrió en la estación 8 en Diciembre con 1,670 org/1000 m³ y la máxima en la estación 11 en Junio con 1,548,094 org/1000 m³ (Fig. 4). En Junio se registró el máximo promedio mensual con 168,093 org/1000 m³), mientras que el mínimo se observó en Octubre con 19,708 org $/ 1000 \mathrm{~m}^{3}$.

Las larvas de peces ocuparon el segundo lugar en abundancia representando el $12.70 \%$ del total del zooplancton recolectado, con una abundancia media de $32,748 \mathrm{org} / 1000 \mathrm{~m}^{3}$. Sus máximas y mínimas densidades se registraron en las estaciones 2 (Junio) y 14 (Octubre) con 399,089 y $215 \mathrm{org} / 1000 \mathrm{~m}^{3}$, respectivamente (Fig. 5). Durante Junio se observaron las máximas densidades promedio $\left(73,076 \mathrm{org} / 1000 \mathrm{~m}^{3}\right)$ y en Octubre las mínimas $\left(1,159 \mathrm{org} / 1000 \mathrm{~m}^{3}\right)$.

Los copépodos calanoides representaron el $12.20 \%$ del total y ocuparon el tercer lugar en abundancia, su abundancia media fue de $31,471 \mathrm{org} / 1000 \mathrm{~m}^{3}$. Estos

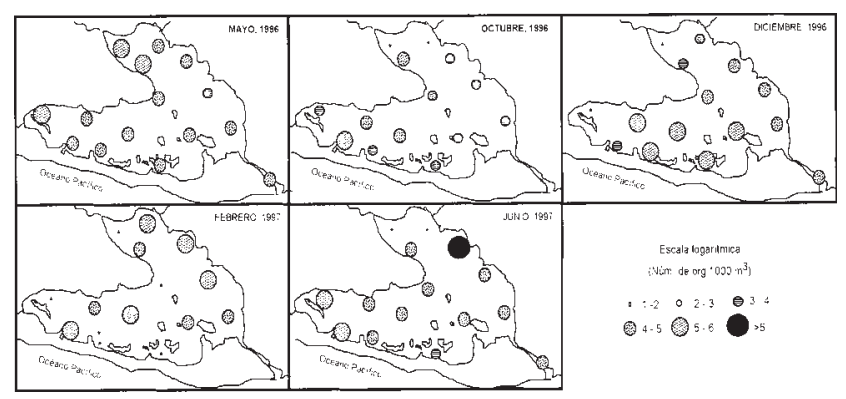

Figura 4. Variación espacio-temporal de la abundancia de larvas de braquiuros en la laguna La Pastoría (* estación sin organismos).

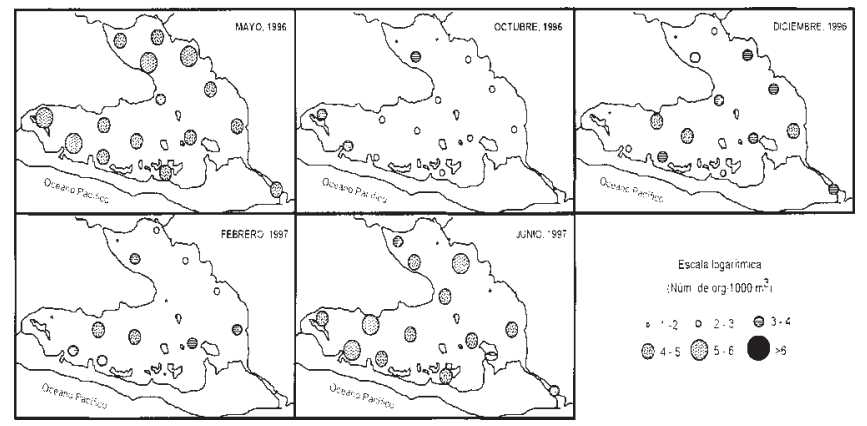

Figura 5. Variación espacio-temporal de la abundancia de larvas de peces en la laguna La Pastoría (* estación sin organismos)

crustáceos presentaron su máxima y mínima abundancia en los meses de Diciembre $\left(87,277\right.$ org/1000 $\left.\mathrm{m}^{3}\right)$ y Octubre $\left(241 \mathrm{org} / 1000 \mathrm{~m}^{3}\right)$, respectivamente. Las estaciones que presentaron las máximas y mínimas densidades de copépodos fueron la estaciones 14 en Diciembre $(610,999$ org/1000 $\mathrm{m}^{3}$ ) y la 5 y 14 en Octubre con 52 y 53 org/1000 $\mathrm{m}^{3}$, respectivamente.

Otros grupos que se observaron con menores abundancias en la laguna fueron los huevos de peces, las larvas de anomuros, los misidáceos y los quetognatos (Cuadro 2).

Las larvas de braquiuros se encontraron distribuidas ampliamente en La Pastoría, registrando sus mayores densidades al norte de la laguna en Mayo y Octubre, al noroeste en Febrero y Junio y al sur en Diciembre. Otros grupos que se encontraron distribuidos en toda la laguna fueron las larvas de peces y los copépodos calanoides, presentando sus máximas abundancias en diferentes regiones de la laguna, aunque observándose ligeramente con mayor frecuencia en las cercanías al canal de comunicación con el mar. Los huevos de peces se encontraron distribuidos en toda la laguna presentando las mayores concentraciones en las estaciones adyacentes al canal que comunica a la laguna con el mar, con excepción de Octubre, mes en el que no se recolectaron en La Pastoría.

No se observaron correlaciones significativas entre las abundancias de ambas lagunas $(\mathrm{r}=0.44, \mathrm{t}=0.85$, ). 
Cuadro 2. Abundancia promedio (org/1000 m3) y relativa (\%) de los grupos zoopláncticos encontrados en la Laguna La Pastoría, Oaxaca, durante el periodo de estudio

\begin{tabular}{|c|c|c|c|c|c|c|c|}
\hline Grupos\Mes & Mayo & Octubre & Diciembre & Febrero & Junio & $\begin{array}{l}\text { Abund. } \\
\text { Prom. } \\
\text { org/1000 m³ }\end{array}$ & $\begin{array}{c}\text { Abund. } \\
\text { Relativa } \\
\%\end{array}$ \\
\hline Larvas de braquiuros & 54508 & 19708 & 97919 & 118591 & 168093 & 91764 & 35.58 \\
\hline Larvas de peces & 72662 & 1159 & 11539 & 5307 & 73076 & 32748 & 12.70 \\
\hline Copépodos calanoides & 12870 & 241 & 87277 & 51128 & 5837 & 31471 & 12.20 \\
\hline Huevos de peces & 55032 & 0 & 1939 & 2883 & 71367 & 26244 & 10.18 \\
\hline Misidáceos & 21891 & 2088 & 44429 & 33481 & 27622 & 25902 & 10.04 \\
\hline Quetognatos & 16581 & 0 & 54577 & 8018 & 8643 & 17564 & 6.81 \\
\hline Ctenóforos & 1946 & 0 & 70468 & 0 & 5 & 14484 & 5.62 \\
\hline Larvas de anomuros & 7158 & 27 & 22345 & 17428 & 4492 & 10290 & 3.99 \\
\hline Medusas & 10463 & 0 & 4869 & 21 & 1304 & 3331 & 1.29 \\
\hline Peneidos & 279 & 9588 & 53 & 98 & 340 & 2072 & 0.80 \\
\hline Larváceos & 98 & 0 & 2977 & 0 & 7 & 616 & 0.24 \\
\hline Bivalvos & 1727 & 0 & 182 & 0 & 7 & 383 & 0.15 \\
\hline Gasterópodos & 170 & 0 & 1967 & 0 & 18 & 431 & 0.17 \\
\hline $\begin{array}{l}\text { Larvas de } \\
\text { estomatópodos }\end{array}$ & 285 & 0 & 806 & 0 & 19 & 222 & 0.09 \\
\hline Cirripedios & 165 & 0 & 141 & 12 & 134 & 90 & 0.04 \\
\hline Ostrácodos & 0 & 0 & 135 & 12 & 183 & 66 & 0.03 \\
\hline Anfípodos & 46 & 0 & 255 & 0 & 2 & 61 & 0.02 \\
\hline Foraminíferos & 0 & 0 & 171 & 83 & 0 & 51 & 0.02 \\
\hline Tanaidáceos & 157 & 7 & 24 & 0 & 7 & 39 & 0.02 \\
\hline $\begin{array}{l}\text { Copépodos } \\
\text { sifonostomatoides }\end{array}$ & 138 & 4 & 5 & 17 & 18 & 36 & 0.01 \\
\hline Poliquetos & 88 & 0 & 24 & 0 & 0 & 22 & 0.01 \\
\hline Taliáceos & 50 & 0 & 30 & 0 & 0 & 16 & 0.01 \\
\hline Isópodos & 7 & 0 & 29 & 0 & 0 & 7 & 0.003 \\
\hline Cumáceos & 28 & 0 & 0 & 0 & 0 & 6 & 0.002 \\
\hline Branquiuros & 0 & 5 & 0 & 6 & 0 & 2 & 0.001 \\
\hline Total & 256348 & 32828 & 402162 & 237084 & 361174 & 257919 & 100.00 \\
\hline
\end{tabular}

Tampoco se observaron diferencias significativas entre las abundancias de cada laguna (Chacahua: $\mathrm{r}=2 \times 10^{-8}, \mathrm{t}=0.83$ x $10^{-9}$; La Pastoría: $\left.r=1 \times 10^{-8}, \mathrm{t}=47 \times 10^{-9}\right)$.

\section{Discusión}

En Chacahua, el período de lluvias se presenta de Mayo a Octubre, sin embargo durante el periodo estudiado éstas se atrasaron, extendiéndose el periodo de sequía hasta principios de Julio. Esto aunado a la poca profundidad y a la nula tasa de intercambio entre la laguna y el mar, originaron una salinidad elevada en Mayo de 1996 (39.5 PSU) y Junio de 1997 (65.3 PSU). Durante el período de sequía (NoviembreAbril), se observaron fuertes variaciones de salinidad en Chacahua, comportándose como un sistema hiposalino (8.2 PSU) debido a los ciclones Boris y Cristina que provocaron intensas lluvias, aún en Noviembre, originando que los ríos se desbordaran y se presentaran escurrimientos hacia la laguna.
Por el contrario, en La Pastoría, la salinidad no presentó variaciones tan marcadas (36.3-37.1 PSU) debido a una menor evaporación, por tratarse de una laguna con mayor profundidad, así como al intercambio de agua que existe entre esta laguna y el mar. Durante el periodo de sequía presentan salinidades similares a las de mar, lo que coincide con lo registrado por SEPESCA (1990) y Contreras et al. (1994) quienes señalan que el impacto ambiental en las lagunas costeras aumenta en la medida de la carencia de la circulación y renovación de sus masas de agua.

Los cambios bruscos de salinidad en Chacahua son debidos a la falta de comunicación con el mar y a los factores anuales de precipitación e insolación, que determinan que esta laguna se caracterice por presentar ambientes de alto contraste estacional en la que sólo permiten el desarrollo de organismos eurihalinos. Es por ello que las comunidades presentan una menor diversidad que en La Pastoría. En esta laguna sucede lo contrario, por tener una comunicación permanente con el mar, existe una 
mayor estabilidad estacional de la salinidad, reflejándose en la estructura de la comunidad.

El comportamiento de la temperatura en el área de estudio coincide con el patrón descrito para la laguna San José Manialtepec, Oaxaca por Contreras y García-Nagaya (1991).

El análisis de la comunidad zooplánctica permitió establecer una marcada diferencia, en cuanto a la composición faunística entre las lagunas de Chacahua y La Pastoría. Los carideos no se registraron en La Pastoría y en Chacahua no se registraron los ctenóforos, los taliáceos, los larváceos, los foraminíferos, las larvas de estomatópodos, los cumáceos y los isópodos. La mayor diversidad zooplánctica registrada en la laguna La Pastoría fue favorecida por el flujo y reflujo de la marea que permitieron el ingreso de estos organismos al interior de la laguna La Pastoría. Por el contrario la menor diversidad observada en Chacahua se debe a la débil influencia marina con su fauna característica, que recibe esta laguna a través del canal del Corral, así como al cierre permanente de la bocabarra. Estos resultados concuerdan con lo encontrado por SEPESCA (1990) quien afirma que la ausencia de intercambio con el mar provoca una desestabilización en el flujo energético de dichos ecosistemas, causando un efecto negativo en la diversidad biológica.

La ocurrencia y abundancia de los organismos meropláncticos en el zooplancton depende de los hábitos de reproducción de los adultos y a la respectiva duración de sus estadios larvales (Gardiner, 1978); estas características son la causa probable de la dominancia de las larvas de braquiuros sobre los otros grupos zoopláncticos encontrados en el complejo lagunar. Además Ruiz (1985) observó en aguas someras de lagunas costeras, bahías, esteros y desembocadura de los ríos que la temporada de reproducción de los braquiuros (jaibas) se lleva a cabo durante la primavera y el verano a temperaturas mayores a $\operatorname{los} 23^{\circ} \mathrm{C}$, cubriendo un período de desove que se prolonga de siete a nueve meses al año, en el cual se registran hasta dos desoves por individuos, generando un número de huevos que se estima entre cien mil y dos millones por desove.

Nuestros resultados coinciden con lo observado por Naranjo (2002) para el estuario interior del Golfo de Guayaquil, donde las larvas de braquiuros son el grupo dominante, presentando abundancias relativas que oscilan entre el 35 y el $85 \%$ del total de zooplancton recolectado.

Muchas especies marinas de importancia pesquera son parcial o totalmente dependientes estuarinas y dependiendo de sus estrategias alimenticias y reproductivas emplean los ambientes lagunar-estuarinos como áreas de reproducción, alimentación, crianza y protección (YáñezArancibia, 1986). Es posible que por esta razón las larvas y huevos de peces fueran tan abundantes en el complejo lagunar, ocupando el segundo y tercer lugar en abundancia. Entre las larvas de peces se encontraron larvas de especies marinas como Centropomus robalito (robalito), Diapterus peruvianus (mojarra aleta amarilla) y Gerres cinereus (mojarra blanca), que conformaron más del $80 \%$ del total de la biomasa capturada del ictioplancton en el trabajo de Aceves (1997). La baja densidad de larvas de peces y la ausencia de huevos de peces observada en el mes de Octubre en ambas lagunas se debe a la finalización del periodo reproductivo de los peces, ésto coincide con lo observado por Aceves (1997). Además Ruíz (1985) señaló que el periodo de desove de estas especies se presenta de Junio a Agosto.

De acuerdo a Day y Yáñez-Arancibia (1982), los copépodos constituyen uno de los grupos que debieron haberse presentado en mayores abundancias en estos ambientes lagunar-estuarinos. Ellos señalan que las poblaciones de copépodos son generalmente el componente más abundante de cualquier muestra de zooplancton, constituyendo del 70 al $90 \%$ de la comunidad zooplánctica. Sin embargo, en este estudio se observó que en La Pastoría los calanoides sólo aparecieron con el $11.3 \%$, mientras que en Chacahua con el $0.83 \%$ y el $6.7 \%$ del total para el complejo lagunar. Castellanos (obs. personal) observó que en arrastres realizados en la Bahía de Chetumal con red de $0.33 \mathrm{~mm}$ de abertura de malla los copépodos representaban el $90.33 \%$ del total del zooplancton. Asimismo, en la Laguna de Términos (Monroy, 1987) y en la laguna Bojorquez (ÁlvarezCadena y Segura-Puertas, 1997) los copépodos constituyen el grupo dominante. Esta diferencia posiblemente se debe a que con la red con abertura de malla de $0.50 \mathrm{~mm}$ se están submuestreando algunos organismos del zooplancton. De esta forma se explica que los resultados obtenidos en este trabajo sean similares a los de la Bahía de Chetumal (Castellanos, 1992; Gasca y Castellanos, 1993), en donde se registró que las larvas de decápodos, los huevos y larvas de peces fueron los grupos dominantes en arrastres realizados con una red con abertura de malla de $0.50 \mathrm{~mm}$, en tanto que los copépodos sólo representaron el $13.7 \%$ del total.

El agua de mar del Océano Pacífico que penetra al complejo lagunar está influenciada por la onda de marea que pasa a través de la bocabarra de Cerro Hermoso y es el principal mecanismo generador de la circulación en la Pastoría (Sanay, 1997). Esto favoreció la presencia de organismos típicamente marinos (medusas, ctenóforos, quetognatos y misidáceos, entre otros) aunque en menores porcentajes.

La presencia de organismos bénticos como poliquetos, cumáceos, tanaidáceos, isópodos, anfípodos, etc., en las muestras puede deberse a la influencia que ejerce la marea sobre la circulación lagunar, a través de la mezcla y turbulencia que provoca y los transporta a la superficie, a los movimientos migratorios y a la escasa profundidad de la laguna.

El aporte de agua marina hacia La Pastoría permite que este sistema tenga un flujo continuo a todo lo largo de la laguna, tanto en condiciones de pleamar como de bajamar, reflejandose ésto en la heterogeneidad de la composición faunística de los taxa y la mayor biomasa zooplánctica, coincidiendo con lo expuesto por Yáñez-Arancibia y Nugent (1977), quienes señalaron que las comunidades varían en su composición y abundancia relativa de especies 
de acuerdo a las condiciones hidrológicas del sistema, encontrando de esta manera en las lagunas especies dulceacuícolas, eurihalinas, estenohalinas y migratorias.

La distribución espacial de la comunidad del zooplancton mostró un patrón relativamente homogéneo en ambas lagunas. La distribución y ausencia de algunos grupos en ciertos meses (como huevos de peces y medusas), podría tener relación con el patrón de circulación del agua durante la época de lluvias lo que produce un intenso acarreo de zooplancton hacia el mar y a la influencia que ejercen las corrientes marinas y dulceacuícolas en el complejo lagunar.

En Chacahua las alteraciones antropogénicas como las obras de infraestructura hidroagrícola para incrementar las superficies de riego, el corte del aporte de agua dulce proveniente del río Verde, están ocasionando una modificación en su estructura (disminución del espejo de agua, mayor azolvamiento, etc.). Esto concuerda con lo observado por Infante (1988) quien señala que el establecimiento y la expansión de las poblaciones humanas afectan con mayor o menor intensidad a los sistemas acuáticos, ya que los productos de las actividades antropogénicas (domésticas y agrícolas) se incorporan a las lagunas ocasionando cambios físicos y químicos, afectando por consiguiente a las comunidades que los habitan.

Por último podemos concluir que la distribución y la concentración del zooplancton se debe principalmente a factores abióticos como la salinidad, así como también a la disponibilidad de alimento, régimen de mareas y época estacional.

\section{Agradecimientos.}

Las muestras fueron recolectadas con del apoyo del proyecto OAX-RNMA-005/96, financiado por el CONACyT. A la Universidad del Mar de Puerto Angel, Oaxaca, por permitir el uso del equipo e instalaciones.

\section{Literatura citada}

Álvarez-Cadena, J. N. y L. Segura-Puertas. 1997. Zooplankton variability and copepod species assemblages from a tropical coastal lagoon. Gulf Research Reports 9: 345-355.

Aceves, G. 1997. Fauna íctica de los sistemas lagunares de Chacahua-La Pastoría y Corralero-Alotengo. In Caracterización ambiental de los recursos naturales de los sistemas lagunares de Chacahua-La Pastoría y Corralero-Alotengo, Vélez, H. (ed.). Informe Técnico Parcial (Diciembre 1998). RNA, Oaxaca. 15 p.

Boltovskoy, D. 1981. Atlas del zooplancton sudoccidental y métodos de trabajo con el zooplancton marino. Inatituto Nacional de Investigación y Desarrollo Pesquero, Mar del Plata, Argentina, $936 \mathrm{p}$.

Castellanos, I. A. 1992. Distribución y abundancia del zooplancton en la Bahía de Chetumal, Q. Roo (Agosto, 1990-Junio, 1991). Tesis profesional, InstitutoTecnológico de Chetumal, Chetumal, Q. Roo, México. 49 p + 29 anexos.
Contreras, E. F. 1993. Ecosistemas costeros mexicanos. CONABIO-UAMI, México, 492 p.

Contreras, E. F. y A. García-Nagaya. 1991. Hidrología, nutrientes y productividad primaria en la laguna San José Manialtepec, Oaxaca, México. Hidrobiológica 1: 65-72.

Contreras, E. F., O. Castañeda, A. García y M. A. Pérez. 1994. Las lagunas costeras. In Riqueza y pobreza de las costas de Chiapas y Oaxaca, Toledo, A. (Comp.). CECODES, México, p. 129-178.

Day, J. y A. Yáñez-Arancibia. 1982. Coastal lagoons and estuaries: ecosystem approach. Ciencia Interamericana 22: 11-26.

Gardiner, M. 1978. Biología de los Invertebrados. Barcelona, España. 940 p.

Gasca, R. y I. Castellanos. 1993. Zooplancton de la Bahía de Chetumal, Mar Caribe, México. Revista de Biología Tropical 41: 619-625.

Imasa SA. de C.V. 1995. Construcción de escolleras de concreto simple y dragado del canal de comunicación en Chacahua, Oaxaca. Manifestación de impacto ambiental. Dirección General de Infraestructura Flota Pesquera, Secretaría de Pesca, México. 256 p.

Infante, A. 1988. El plancton de aguas continentales. Monografía No. 33. Serie de Biología. OEA. Washington. $130 \mathrm{p}$.

Monroy, C. 1987. Análisis de la comunidad zooplanctónica en el Complejo Lagunar de Términos, Campeche, durante 1982. Tesis profesional. Universidad Autónoma de Baja California, México. 34p.

Naranjo, C. 2002. Zooplancton en el estuario interior de Guayaquil, durante 2001. Acta Oceanográfica del Pacífico, Instituto Oceanográfico de la Armada 11: 101112.

Odum, E. P. 1972. Ecología. Interamericana, México. 639p.

Raymont, J. E. G. 1983. Plankton and productivity in the oceans. Vol. II. Zooplankton. Pergamon, Oxford. 824p.

Rodríguez, E. 1998. Variabilidad estacional de la hidrología y transporte litoral en la laguna de Chacahua, Oaxaca. Tesis profesional. Universidad Nacional Autónoma de México, México, D.F. 64 p.

Ruíz, M. F. 1985. Recursos pesqueros de las costas de México. Limusa. 208 p.

Sanay, R. 1997. Simulación de la circulación en el Sistema Lagunar Chacahua-La Pastoría, Oaxaca. Tesis de Maestría. Universidad Nacional Autónoma de México, México, D.F. 107 p.

Sepesca. 1990. Bases para el ordenamiento costero-pesquero de Oaxaca y Chiapas. Secretaría de Pesca, México. 219 p.

Smith, D. L. 1977. A guide to marine coastal plankton and invertebrate larvae. Kendall/Hunt, Iowa, USA. $161 \mathrm{p}$

Smith, P. E. y S. L. Richardson. 1979. Técnicas modelo para la prospección de huevos y larvas de peces pelágicas. FAO. Documento Técnico Pesca 175: 1-107 
Tait, V. 1987. Elementos de ecología marina. Editorial Acribia, Zaragoza, España. 446 p.

Tregouboff, G. y M. Rose. 1957. Manuel de Planctonologie Mediterraneénne. Tomo I. Centre National de la Recherche Scientifique, Paris. 202 p.
Yáñez-Arancibia, A. 1986. Ecología de la zona costera. Análisis de siete tópicos. A.G.T. Editor, México. 189p. Yáñez-Arancibia, A. y R. Nugent. 1977. El papel ecológico de los peces en estuarios y lagunas costeras. Anales del Centro de Ciencias del Mar y Limnología 4: 107-144. 